\title{
Contributors List
}

\section{Florenci V. González Adelantado}

Departament de Química Inorgànica i

Orgànica

Universitat Jaume I

Avda. Sos Baynat, s/n

Castelló 12071, Spain,

E-mail: fgonzale@uji.es

\section{Anthony Burke}

Chemistry Department

University of Evora

Rua Romão Ramalho 59

7000-671 Evora, Portugal

E-mail: ajb@uevora.pt

\section{Armando Carlone}

Department of Physical and Chemical

\section{Sciences}

Università degli Studi dell'Aquila, Coppito, via Vetoio

L'Aquila 67100, Italy

E-mail: armando.carlone@univaq.it

\section{Pier Giorgio Cozzi}

Università degli Studi di Bologna

Dipartimento di Chimica "G. Ciamician"

via Selmi 2

40126 Bologna, Italy

E-mail: piergiorgio.cozzi@unibo.it

\section{Yujiro Hayashi}

Department of Chemistry

Graduate School of Science

Tohoku University

Aoba-ku, Sendai 980-8578, Japan

E-mail: yhayashi@m.tohoku.ac.jp

\section{Christoforos Kokotos}

Laboratory of Organic Chemistry

Department of Chemistry
National and Kapodistrian University of

Athens

Panepistimiopolis,

Athens 15771, Greece

E-mail: ckokotos@chem.uoa.gr

\section{Alessandra Lattanzi}

Dipartimento di Chimica e Biologia

"A. Zambelli"

Università di Salerno

via Giovanni Paolo II 132

Fisciano 84084, Italy

Email: lattanzi@unisa.it

\section{Elisabetta Massolo}

Dipartimento di Chimica

Università degli Studi di Milano

Via Golgi, 19

Milano 20133, Italy

E-mail: elisabetta.massolo@unimi.it

\section{Manuel Orlandi}

Dipartimento di Scienze Chimiche

Università degli Studi di Padova

via Marzolo 1, Padova 35131, Italy

E-mail: manuel.orlandi@unipd.it

\section{Alessandra Puglisi}

Dipartimento di Chimica

Università degli Studi di Milano

via Golgi 19

Milano, 20133 Italy

E-mail: alessandra.puglisi@unimi.it

\section{Laura Raimondi}

Dipartimento di Chimica

Università degli Studi di Milano

via Golgi, 19

Milano, Lombardia 20133, Italy

Email: lauramaria.raimondi@unimi.it 
